\title{
A hybrid digital image watermarking scheme incorporating DWT, DFT, DCT, and SVD transformations
}

\author{
Justin Varghese*, Omer Bin Hussain**, T Abdul Razak*** and Saudia Subash**** \\ * Karpagam College of Engineering, Coimbatore, Tamil Nadu, India \\ **College of Computer Science, King Khalid University, Abha, Saudi Arabia \\ ***Jamal Mohammed College, Tiruchirapalli, Tamil Nadu, India \\ **** Centre for Information Technology \& Engineering, Manonmaniam Sundaranar University, India \\ *Corresponding Author: justin_var@yahoo.com
}

$\begin{array}{ll}\text { Submitted } & : 30 / 01 / 2020 \\ \text { Revised } & : 26 / 02 / 2021 \\ \text { Accepted } & : 02 / 06 / 2021\end{array}$

\begin{abstract}
Digital watermarking provides copyright protection and proof of ownership by inserting watermark metadata as owner's identity in digital documents to prevent authenticity and copyright violations. The paper introduces a new hybrid image watermarking scheme by attaching multiple copies of watermarks in carrier image. The new scheme utilizes the advantages of DWT, DFT, DCT, and SVD transformations to offer stable resistance in protecting watermark contents from various external attacks. The proposed scheme uses Haar wavelet, Fourier, Onion Peel Decomposition, DCT, zigzag ordering, and SVD transformations to decompose the carrier image into four levels to maintain imperceptibility in the watermarked images. The algorithm attaches replicas of watermark frequency blocks in all frequency components of host image to provide better robustness against external deprivations in watermarked images. The proposed algorithm also provides the increased probability of extracting at least one undamaged replica of watermark even when other frequencies are damaged by external attacks. The improved experimental results of the proposed scheme in terms of visual analysis and quantitative metrics on different images with different experimental setup demarcate that the proposed watermarking scheme provides stable performance in generating better watermarked images. It is experimentally found that the new scheme produces high quality watermarked images with an average of 7.62\% lesser Mean Absolute Error (MAE) and increased Peak Signal to Noise Ratio (PSNR), Mean Structural Similarity Index Measure (MSSIM), and Feature Similarity Index Measure (FSIM) of 5.02 $\%, 4.37 \%$, and $2.37 \%$, respectively, than the next best algorithms when simulated with 20 sets of watermark and cover images. The watermark images extracted by the proposed algorithm from extremely distorted watermarked images are with better visual and objective values than other methods used in the comparative study. Simulation analysis on 20 sets of watermark and cover images with 30 types of potential attacks reveals that the extracted watermark images through the proposed scheme produces an average of 5.62\%, 6.37\%, and 5.75\% improved Pearson Correlation Coefficients (PCC), Number of Changing Pixel Rate (NPCR), and the Unified Averaged Changed Intensity (UACI) values, respectively, than the next best algorithms used in the comparative study.
\end{abstract}

Keywords: Copyright protection; Discrete cosine transform; Discrete wavelet transform; Watermarking; Discrete fourier transform; Information security. 


\section{INTRODUCTION}

The evolution of communication technologies and its widespread use have made the communication of digital documents faster, convenient, and easily accessible (Borra et al., 2018). It has also led to additional vulnerabilities related to counterfeiting, authenticity, integrity, copyright violation, source tracking, broadcast monitoring, ID card security, fraud and tamper detection, content management, etc. Watermarking techniques are developed for maintaining intellectual property/copyright/ownership rights of documents in a cost-effective manner by embedding visible/invisible watermarks as meta-data in the host/owner's documents as authentication identities (Nematollahi et al., 2017; Umamageswari et al., 2014). Digital watermarking techniques help perform transaction tracking, broadcast monitoring, device control, forensics, and content filtering.

Based on the processing in which watermarks are attached, watermarking techniques are grouped into spatial and frequency methods. Spatial domain methods insert watermarks in Least Significant Bit (LSB), altering pixel values of the carrier image (Hussain et al., 2019). Frequency domain methods attach watermark contents in the frequencies of host image rather than attaching directly to the image contents. These methods make the opportunity for selecting suitable frequencies to attach watermark contents, so that the changes due to watermark embedding are not much visible in the host image. Since watermarking techniques in frequency domain embed watermark contents in different frequencies of cover image, these methods show high robustness in protecting watermark from potential attacks than spatial domain techniques. This study proposes a new hybrid watermarking scheme by combining the advantages of DWT, DFT, DCT, and SVD transformations and by attaching multiple copies of watermark contents in carrier image.

\section{RELATED WORKS AND REVIEW}

Frequency domain transformations such as DWT, DFT, and DCT decompose the images into different frequency levels and thereby offer watermarking techniques to show better imperceptibility, robustness, security, and capacity by selecting suitable frequency bands to attach watermark contents. Exploiting the advantages of these transformations while embedding watermark contents in the host image, many algorithms (Li et al., 2018; Mehta et al., 2018; Varghese et al., 2014; Varghese et al., 2015; Yadav et al., 2018; Sharma et al., 2017; Su et al., 2019; Ganic et al., 2004) evolved by incorporating different frequency transformations. Li J. et al. (2018) proposed a Quaternion Hadamard Transform (QHT) based image watermarking scheme. The algorithm creates blocky effects in the watermarked images since it adopts block based processing. The watermarking scheme introduced by Mehta R. et al. (2018) uses Genetic Algorithm (GA) and Lagrangian Support Vector Regression (LSVR) in wavelet domain to select specific blocks of host image for attaching watermark contents according to specific regression function. But the algorithm takes more computation time to optimize regression function using GA and LSVR. Varghese J. et al. (2015) attached watermark contents in Discrete Cosine Transform (DCT) domain by selecting suitable carrier blocks according to human visual characteristics based on the entropy of different carrier and watermark frequency blocks. B. Yadav (2018) attached watermark contents directly to the singular values of third level Discrete Wavelet transform (DWT). But the extracted watermarks often get destroyed due to external potential attacks since the algorithm attaches watermark contents in the low frequencies alone. Sharma et al. (2017) introduced an LSB based watermarking technique that inserts the watermark into the carrier image using LSB and Multiple-Parameter Discrete Fractional Fourier transform (MPDFRFT) for improving robustness. But the execution time required for this proposal is very high. Su Q. et al. (2019) introduced spatial domain watermarking procedure by embedding watermark contents in all pixels of the carrier image by quantization; but the watermarked results reflect weak robustness to potential attacks. Hsu, C. S. et al. (2019) proposed a watermarking scheme specifically for resisting cropping attacks. Wang, C. et al. (2019) used Ternary radial harmonic Fourier moments to hide watermark contents. Najafi, E. \& Loukhaoukha, K. (2019) introduced a watermarking scheme by utilizing the advantages of SVD and contourlet 
transform. Hu, H. T. et al. (2020) used mixed modulation while attaching watermark contents in carrier images. Thakur, S. et al. (2020) incorporated DWT and SVD hamming code and chaotic encryption to perform encryption during watermark embedding. Ko, H. J. et al. (2020) used interblock correlation to find suitable carrier blocks for attaching watermark contents.

Although these frequency domain methods show improvements in watermarking than spatial domain methods, they find difficulties in retrieving valid watermark contents especially when potential attacks damage the frequencies, where the watermarks are attached. These disadvantages of single copy watermark attachment in specific frequencies are being taken up by other schemes (Ganic et al., 2004; Sverdlov et al., 2006; Run et al., 2012; Varghese et al., 2016) to attach multiple watermark replicas in all frequencies to provide better robustness against external deprivations in watermarked images. Watermarks attached in specific frequencies have specific resistance to specific group of attacks, and it varies according to the frequency, where the watermark contents are attached. Hence, if watermark replicas are attached in all frequencies, it is difficult to eliminate or erase the watermark from different frequencies. By attaching replicas of watermark contents at different frequencies of host image, these algorithms always hold higher probability to extract some valid watermark replicas from some host frequencies even when other frequencies are damaged by external attacks. Although these algorithms with multiple watermark replica attachment offer many advantages, their robustness largely depends on the frequency transformations, where different transformations offer different levels of robustness against different external attacks.

When comparing the advantages and disadvantages of major frequency transformations in the field of watermarking, it is identified that DWT provides better spatial-frequency localization of signals due to its inherent multiresolution based basis functions (Poljicak et al., 2011). Due to the wide applications of DCT in JPEG based compression schemes, DCT provides better robustness towards JPEG compression with medium level of energy compaction. Due to the strong sinusoidal properties, Discrete Fourier Transform (DFT) provides robust energy compaction with geometrical invariance properties (Poljicak et al., 2011). Hence, many watermarking schemes (Singh, 2017; Navas et al., 2008) came up by combining these transformations in watermarking to offer collective resistance towards various potential attacks. But these algorithms suffer the disadvantages of single copy watermark attachment in specific frequencies. Further, these algorithms though combine DCT, DWT, and SVD transformations, they did not take the advantages of DFT or collecting similar frequencies from different subbands to protect watermark contents from external potential attacks.

\section{THE WATERMARKING SCHEME}

The new hybrid watermarking algorithm exploits the advantages of DWT, DFT, DCT, and SVD transformations and offers stable resistance in protecting watermark contents from different external attacks without compromising image fidelity and robustness aspects of image watermarking. Since the watermarks attached in specific frequencies have specific resistance to specific group of attacks, the proposed algorithm attaches watermark contents to all frequencies of carrier image. The new hybrid watermarking scheme is outlined in Fig. 1. The new hybrid watermarking algorithm demarcated in Fig. 1 attaches copies of watermark in all frequencies that have extended probability of extracting a valid watermark even from highly impinged watermarked images by external potential attacks than other competing schemes. 


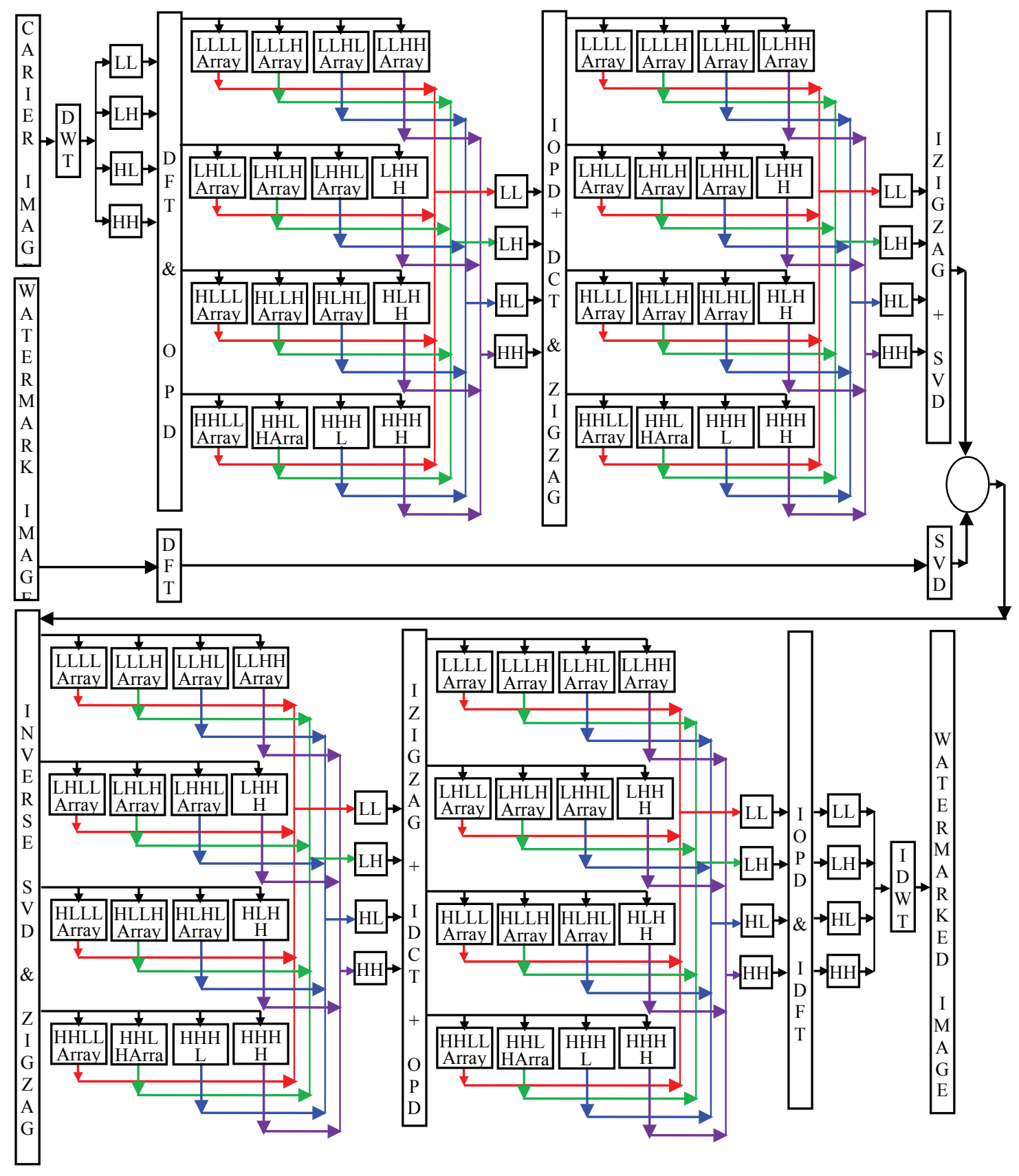

Figure 1. Schematic diagram of new watermarking scheme 
At each level of decomposition by different frequency transformations, the new scheme combines similar frequencies from all decomposed frequency subbands before applying next level of decomposition by a different frequency transformation. By combining similar frequency subbands at different levels by different frequency transformations as in Fig. 1, the algorithm provides more robust platform for attaching watermark components. The scheme starts its watermarking process by decomposing the host image with DWT. Further, these algorithms though combine DCT, DWT, and SVD transformations, they did not take the advantages of DFT.

The subsequent sections detail watermark embedding and retrieving stages.

\section{The watermark embedding}

The new scheme undergoes four levels of decomposition to maintain imperceptibility qualities in the watermarked images. As in Fig. 1, the algorithm applies Haar wavelet transform and converts the carrier image into Low-Low (LL), Low-High (LH), High-Low (HL), and High-High (HH) frequencies. These frequencies are transformed to second-level frequency decomposition by applying DFT. The Onion Peel Decomposition (OPD) operation (Varghese et al., 2016) is applied to these individual frequencies for converting the frequency blocks to corresponding one dimensional array.

From these individual blocks, the corresponding LL, LH, HL, and HH components are separately collected, and the combined arrays are reshaped back to individual blocks by performing Inverse Onion Peel Decomposition (IOPD) operation. As the third level of frequency decomposition, these second-level frequency blocks are further subjected to DCT, and the resultant frequency blocks are reshaped into one-dimensional form by performing zigzag ordering. The LL, LH, HL, and HH components of these individual frequency arrays are separately collected and combined, and the resultant arrays are reshaped back to individual frequency blocks by performing inverse zigzag operation. SVD is finally applied to these frequency blocks as the fourth level frequency decomposition, and the singular values of carrier frequency blocks are combined with singular values of the replicas of watermark frequencies. The algorithm is detailed in the subsequent steps.

Step 1: Since DWT shows spatial-frequency characteristics with localization properties in frequency and time domains, it maintains visible transparency than other transformations (Poljicak, A. et al., 2011) by producing high visual qualities in the watermarked images. DWT also offers multiresolution analysis with strong resistance towards noise and contrast based attacks. With the aim of achieving these advantages in the watermarked images, the algorithm applies DWT on carrier image $C$ to create LL, LH, HL, and HH frequency blocks.

Step 2: Fourier transform has strong geometrical invariance properties with high energy compaction capabilities, and it outperforms DWT in protecting watermark contents in case of geometric based external attacks (Poljicak et al., 2011). For incorporating these advantages, DFT is applied as a second-level decomposition on LL, $\mathrm{LH}, \mathrm{HL}$, and HH subbands of DWT. DFT is performed on watermark image, $\mathrm{CW}$ for generating the watermark frequency, $W$.

Step 3: OPD is a circular traversal applied on Fourier transformed images to decompose frequencies into onedimensional array, such that the traversal sweeps all frequency components in the order starting from extremely high top left corner to the extremely low Direct Current (DC) frequencies. The algorithm of OPD and its illustration are found in the author's previous work (Varghese et al., 2016). OPD is performed on all Fourier transformed twodimensional frequency block images to reshape two-dimensional blocks to one-dimensional format devoid of changing the frequency order (Navas et al., 2008). For notational convenience, $F_{i} \forall i=1,2, \ldots 4$ denote the Fourier transformed frequency blocks and $\left[F L L_{i} F L H_{i} F H L_{i} F H H_{i}\right], \forall i=1,2, \ldots 4$ denote the resultant blocks of OPD. 
Step 4: The proposed scheme groups all similar frequencies from $\left[F L L_{i} F L H_{i} F H L_{i} F H H_{i}\right], \forall i=1,2, \ldots 4$ arrays as

$$
\begin{aligned}
& F B l_{1}={ }_{i=1}^{4} F L L_{i} \\
& F B l_{2}={ }_{i=1}^{4} F L H_{i} \\
& F B l_{3}={ }_{i=1}^{4} F_{H} \\
& F_{i}={ }_{i=1}^{4} \mathrm{FHH}_{i}
\end{aligned}
$$

Here, $\mathrm{FBl}_{1}, \mathrm{FBl}_{2}, \mathrm{FBl}_{3}$ and $\mathrm{FBl}_{4}$, respectively, denote LL, LH, HL, and HH components of OPD arrays.

Step 5: As the next step, inverse OPD is applied on these one-dimensional arrays $F L L, F L H, F H L$ and $F H H$ to restructure them into two-dimensional blocks $B_{i}, \forall i=1,2, \ldots 4$ as

$$
B_{i}=\operatorname{IOPD}\left(F B l_{i}\right)
$$

Step 6: DCT shows better robustness against JPEG compression and block processing based external attacks (Singh et al., 2017), and, hence, DCT is applied as the third level frequency decomposition of the proposed scheme to further decompose the frequency contents of each $B_{i}$ to $D_{i}, \forall i=1,2, \ldots 4$.

Step 7: Further, these two-dimensional DCT blocks $D_{i}$ are converted into one dimension by applying zigzag ordering to collect similar frequencies in the third level decomposition as one-dimensional arrays $\left[D L L_{i} D L H_{i} D H L_{i} D H H_{i}\right], \forall i=1,2, \ldots 4$.

Step 8: In order to effectively collect the similar frequencies from different frequency blocks in the third level of decomposition, the proposed scheme groups all similar frequencies from arrays $\left[D L L_{i} D L H_{i} D H L_{i} D H H_{i}\right]$, $\forall i=1,2, \ldots 4$ such that

$$
\begin{aligned}
& \mathrm{DBl} l_{1}={ }_{i=1}^{4} D L L_{i} \\
& \mathrm{DBl} l_{2}={ }_{i=1}^{4} D L H_{i} \\
& \mathrm{DBl}_{3}={ }_{i=1}^{4} \mathrm{DHL} L_{i} \\
& \mathrm{DBl}_{4}={ }_{i=1}^{4} \mathrm{DHH}_{i}
\end{aligned}
$$

Here, $D B l_{1}, D B l_{2}, D B l_{3}$ and $D B l_{4}$, respectively, denote LL, LH, HL, and HH components of zigzag ordered arrays. 
Step 9: In order to apply SVD operation in the proposed scheme, inverse zigzag operation is applied on $D B l_{1}$, $\mathrm{DBl}_{2}, \mathrm{DBl}_{3}$ and $\mathrm{DBl}_{4}$, respectively, to restructure them into two-dimensional blocks $D B_{i}, \forall i=1,2, \ldots 4$ as

$$
D B_{i}=\operatorname{izigzag}\left(D B l_{i}\right)
$$

Step 10: The singular values of SVD are less affected with external perturbations of cover image, and small changes in singular value contents do not much disturb the image fidelity of the host image (Borra et al., 2011). SVD is individually applied on DWT-DFT-DCT frequency blocks $D B_{i}$ and watermark frequency block, $W$ to combine the watermark contents in the singular values of carrier frequencies. The SVD operations are given by

$$
\begin{aligned}
& D B_{i}=U_{i} S_{i} V_{i}^{T} \quad \forall 1 \leq i \leq 4 \\
& W=\stackrel{w}{U} \stackrel{w}{S} \stackrel{w}{V}^{T}
\end{aligned}
$$

Here, ' $T$ ' is the matrix transpose operation, while $U_{i}, V_{i}, \stackrel{w}{U}$ and $\stackrel{w}{V}$ are unitary matrices of SVD. Further, $S_{k}$ and $\stackrel{w}{S}$ are diagonal matrices with positive real numbers that denote singular value matrices of carrier and watermark frequency blocks, respectively.

Step 11: Once the algorithm has reached four levels of frequency decomposition, the watermark attaching process is performed by combining the cover and watermark singular values. The operation is defined as

$$
\tilde{S}_{i}=S_{i}+\alpha_{i} \stackrel{w}{S} \quad \forall 1 \leq i \leq 4
$$

where $\alpha_{i}$ denotes the strength parameter, while $\tilde{S}_{i}$ is the watermarked singular values for the $i^{\text {th }}$ carrier frequency block. The strength parameter controls the amount of watermark contents in the watermarked images, and its value highly depends on the application.

Step 12: Once the watermark embedding process is over, the algorithm applies inverse SVD, zigzag ordering, inverse frequency rearrangement, inverse zigzag ordering, inverse DCT, OPD, inverse frequency rearrangement, inverse OPD, inverse DFT, and inverse DWT operations for reconstructing watermarked image, $\tilde{C}$ by performing in order the inverse processes from step 10 to 1.

\section{The watermark extraction}

The section details various steps involved in extracting watermark contents from watermarked images. The watermark extraction stage follows the same steps as the embedding scheme but extracts the watermark contents from watermarked images. The subsequent steps detail the watermark extraction process.

Step 1: As the first step of watermark extraction, the algorithm applies steps 1 to 10 of watermark embedding scheme on watermarked image $C$ with possible attacks to extract the singular value matrix, $S_{i}$ from DWT-DFTDCT frequency blocks $D B_{i}$. The SVD operation is given by

$$
\tilde{D B_{i}}=\tilde{U}_{i} \tilde{S}_{i} \tilde{V}_{i}^{T} \quad \forall 1 \leq k \leq 4
$$

where ' $T$ ' is the matrix transpose operation, and $\tilde{U}_{i}$ and $\tilde{V}_{i}$ are unitary matrices of SVD. 
Step 2: Once the algorithm has reached four levels of frequency decomposition, the watermark extraction process is performed for extracting watermark singular values. The operation is defined as

$$
{\stackrel{W}{W^{*}}}_{i}=\frac{\left(\tilde{S}_{i}-S_{i}\right)}{\alpha_{i}} \quad \forall 1 \leq i \leq 4
$$

where $\alpha_{i}$ denotes strength parameter, and $\tilde{S}_{i}$ is the watermarked singular values of the $i^{\text {th }}$ frequency block.

Step 3: The algorithm applies inverse SVD to the individual watermark singular values and regenerates the watermark frequency blocks using the extracted singular value replicas as

$$
\tilde{W}_{i}=\stackrel{w}{U} \stackrel{\mathrm{W}^{*}}{S_{i}} \stackrel{w^{\prime}}{V}
$$

where $\alpha_{i}$ denotes strength parameter, and $S_{i}$ is the watermarked singular values of the $i^{\text {th }}$ frequency block.

Step 4: Finally, the watermark image replicas, $C W_{i}$ are being regenerated by performing the inverse Fourier transform on $W_{i}$.

Since the proposed scheme combines DWT, DFT, DCT, and SVD transformations in its four level frequency separation and similar frequency collection processes, it not only has the advantage of providing high quality watermarked outputs, but also offers better resistance towards noise, contrast, geometric, JPEG compression, and block based external attacks. Further, by attaching multiple watermark replicas in all frequencies of carrier images, the proposed algorithm provides better chance for extracting at least one undamaged copy of watermark replica even when the potential attack affects other frequencies of watermarked image.

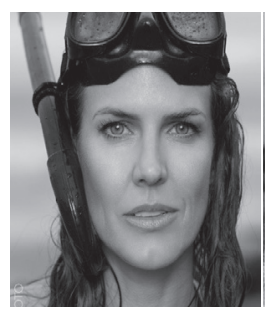

(a) Scuba

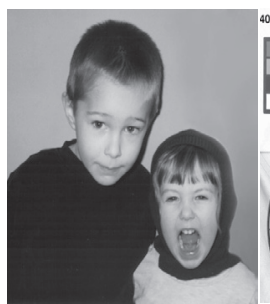

(g) Boys

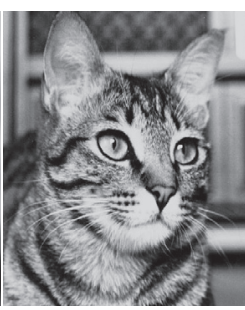

(b) Cat

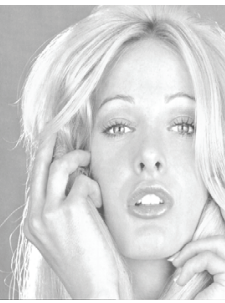

(c) Tiffany

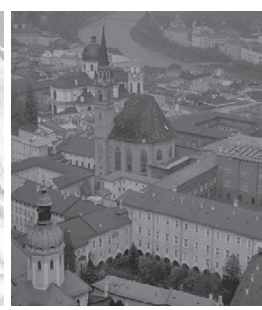

(d) Houses

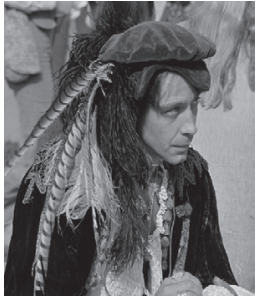

(e) Man

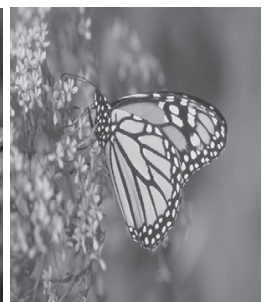

(j) Butterfly

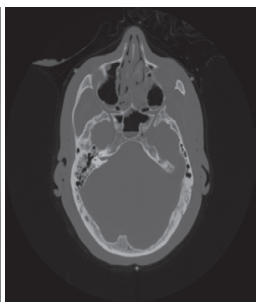

(k) CTScan

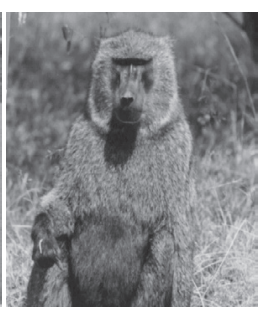

(f) Baboon

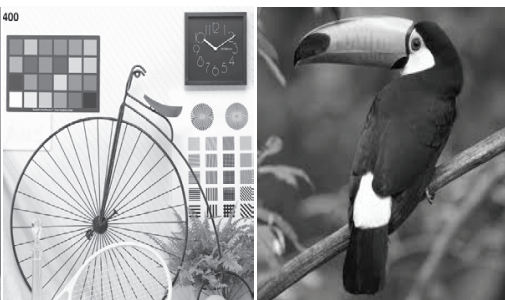

(h) Bicycle

(i) Hornbill

Figure 2. Test images used for experimental analysis as cover $((a)-(f))$ and watermark $((g)-(1))$ images. 


\section{EXPERIMENTAL SIMULATIONS AND ANALYSIS}

The experimental simulations presented in this manuscript are made using MATLAB R2014 software in an Intel $3.3 \mathrm{GHz}$ i3 processor 3220 with 4 GB RAM on a 64-bit Windows operating system. Further, the MATLAB code of the new scheme used in this analysis will be made available through https://in.mathworks.com/matlabcentral/profile/authors/17446231 once the paper is published. The image fidelity and robustness of the new watermarking algorithm are analyzed with wide variety of images, of which Scuba, Cat, Tiffany, Houses, Man, Baboon, Boys, Bicycle, Hornbill, Butterfly, CT Scan, and Clown images are discussed in this paper. Fig. 2 shows the test images used in this study. The effectiveness of new watermarking scheme with respect to image fidelity and robustness is performed with Ganic E. et al. (2004), Sverdlov A. et al. (2006), and Varghese J. et al. (2016) algorithms that embed similar replicas of watermark images with similar strength. These algorithms are selected for comparison since they attach the same number of watermark replicas in carrier image frequencies with similar payload. The strength parameter $\alpha_{i}$ is adjusted for all comparative algorithms for setting equal payload in terms of average pixel wise bitrate of different algorithms.

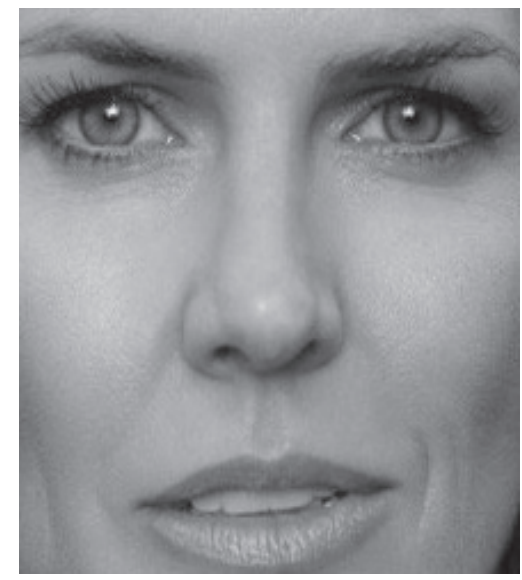

(a) Original Image

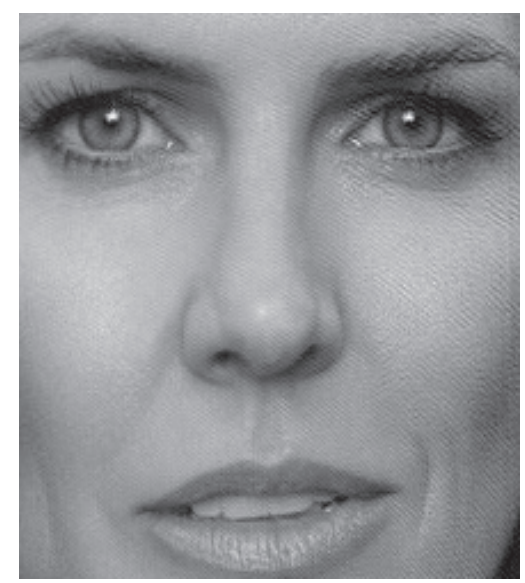

(d) Sverdlov, A. et al.

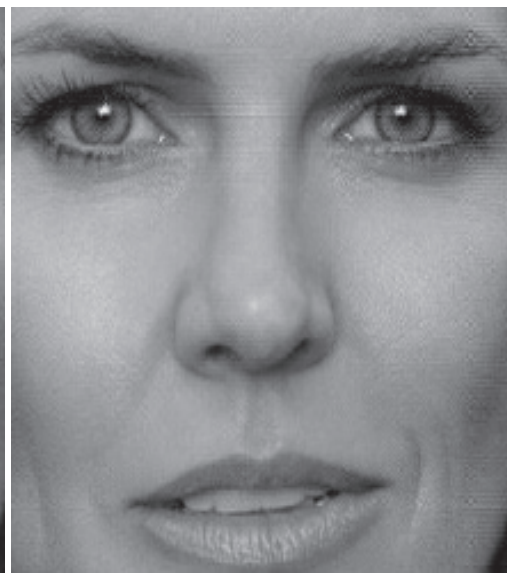

(b) Run RS et al.

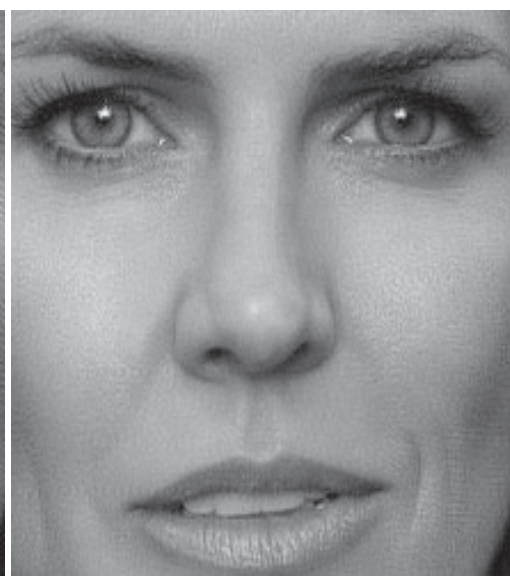

(e) Varghese J et al.

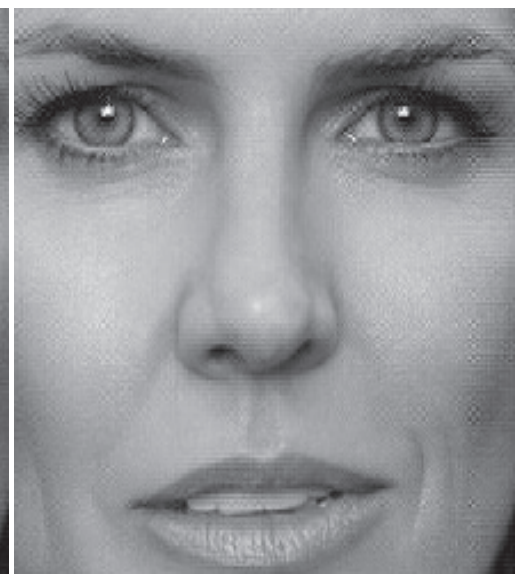

(c) Ganic E. et al.

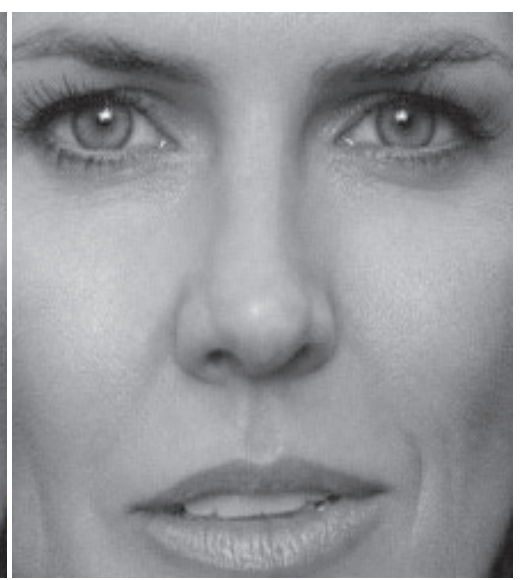

(f) Proposed Algorithm

Figure 3. Cropped versions of watermarked Scuba images of different algorithms with Boys image as watermark. 


\begin{tabular}{|c|c|c|c|c|c|c|c|}
\hline \multirow{2}{*}{$\begin{array}{c}\text { Quantitative } \\
\text { Metric }\end{array}$} & \multirow{2}{*}{$\begin{array}{l}\text { Carrier } \\
\text { Image }\end{array}$} & \multirow{2}{*}{$\begin{array}{l}\text { Watermark } \\
\text { Image }\end{array}$} & \multicolumn{5}{|c|}{ Algorithms } \\
\hline & & & $\begin{array}{c}\text { Run R S } \\
\text { et al. }\end{array}$ & $\begin{array}{c}\text { Ganic E } \\
\text { et al. }\end{array}$ & $\begin{array}{l}\text { Sverdlov A } \\
\text { et al. }\end{array}$ & $\begin{array}{c}\text { Varghese J } \\
\text { et al. }\end{array}$ & $\begin{array}{c}\text { Proposed } \\
\text { Scheme }\end{array}$ \\
\hline \multirow{6}{*}{ MAE } & Scuba & Boys & 6.0163 & 6.7580 & 6.8391 & 4.7128 & 3.6132 \\
\hline & Cat & Bicycle & 10.8120 & 12.8033 & 12.8354 & 6.9695 & 6.7064 \\
\hline & Tiffany & Hornbill & 6.1358 & 5.3390 & 6.3186 & 4.4338 & 4.1275 \\
\hline & Houses & Butterfly & 6.4814 & 7.4460 & 7.1886 & 4.4973 & 4.3438 \\
\hline & Man & CTScan & 2.5933 & 2.7743 & 2.7913 & 1.3169 & 1.7914 \\
\hline & Baboon & Clown & 5.1922 & 6.5835 & 5.5203 & 2.8500 & 3.0553 \\
\hline \multirow{6}{*}{ PSNR } & Scuba & Boys & 29.7690 & 26.6351 & 26.4987 & 34.4806 & 35.4762 \\
\hline & Cat & Bicycle & 25.6048 & 22.3475 & 22.2479 & 30.1932 & 30.2241 \\
\hline & Tiffany & Hornbill & 30.4167 & 28.0459 & 27.8115 & 35.3083 & 35.4112 \\
\hline & Houses & Butterfly & 30.2567 & 27.2532 & 27.0805 & 35.1154 & 35.1696 \\
\hline & Man & CTScan & 37.6932 & 35.3099 & 34.9682 & 43.2911 & 43.2549 \\
\hline & Baboon & Clown & 31.9515 & 29.0001 & 28.8100 & 36.8875 & 36.7502 \\
\hline \multirow{6}{*}{ MSSIM } & Scuba & Boys & 0.9327 & 0.9999 & 0.9999 & 0.9814 & 0.9994 \\
\hline & Cat & Bicycle & 0.9629 & 0.9998 & 0.9998 & 0.9873 & 0.9933 \\
\hline & Tiffany & Hornbill & 0.9927 & 1.0000 & 0.9992 & 0.9871 & 0.9973 \\
\hline & Houses & Butterfly & 0.9883 & 0.9999 & 0.9999 & 0.9899 & 0.9983 \\
\hline & Man & CTScan & 0.9692 & 0.9972 & 0.9962 & 0.9972 & 0.9926 \\
\hline & Baboon & Clown & 0.9931 & 0.9753 & 0.9881 & 0.9950 & 0.9988 \\
\hline \multirow{6}{*}{ FSIM } & Scuba & Boys & 0.9957 & 0.9685 & 0.9973 & 0.9954 & 0.9984 \\
\hline & Cat & Bicycle & 0.9938 & 0.9571 & 0.9939 & 0.9957 & 0.9978 \\
\hline & Tiffany & Hornbill & 0.9947 & 0.9999 & 0.9911 & 0.9964 & 0.9968 \\
\hline & Houses & Butterfly & 0.9936 & 1.0000 & 0.9934 & 0.9971 & 0.9995 \\
\hline & Man & CTScan & 0.9991 & 1.0000 & 0.9994 & 0.9996 & 0.9998 \\
\hline & Baboon & Clown & 0.9959 & 1.0000 & 0.9980 & 0.9983 & 0.9991 \\
\hline
\end{tabular}

Image fidelity measures the similarity between watermarked and original images, while robustness is the assessment of ability of an algorithm to protect the attached watermarks even in case of extreme potential attacks. An effective watermarking algorithm needs to trade off between robustness and image fidelity. Image fidelity of watermarked images generated by comparative algorithms is evaluated in terms Mean Absolute Error (MAE), Peak Signal to Noise Ratio (PSNR), Mean Structural Similarity Index Measure (MSSIM), and Feature Similarity Index Measure (FSIM). The formulations of PSNR, MSSIM, and FSIM are as denoted in similar papers (Ganic et al., 2004; Sverdlov et al., 2006; Run et al., 2012; Varghese et al., 2016). 


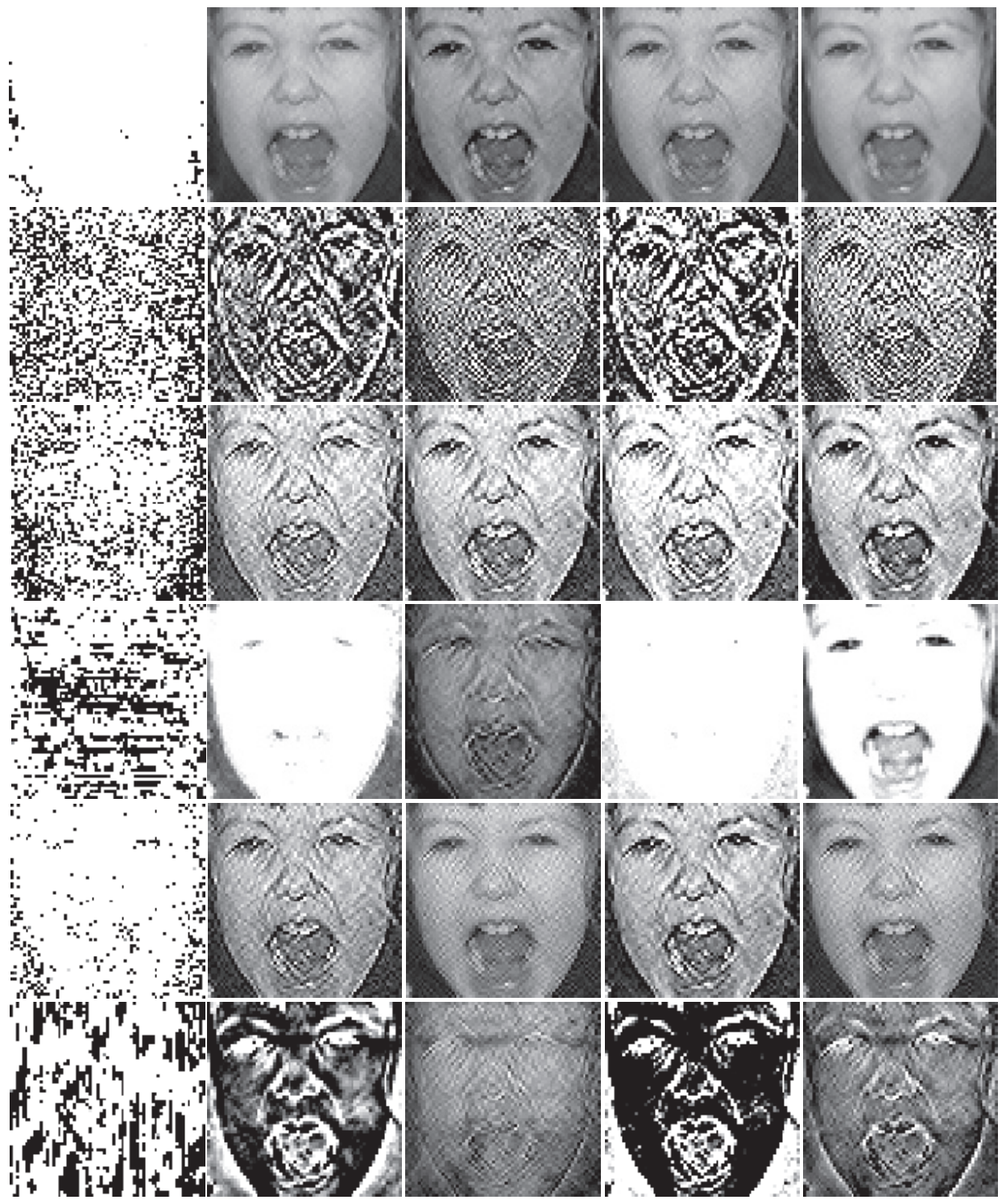

Figure 4. Cropped watermark extracted by comparative schemes from watermarked Scuba image affected by different external attacks: Rows 1-6 are Attacks (Row 1: JPEG80, Row 2: Salt\&Pepper05, Row 3: Gaussian Noise02, Row 4: Avg Filter,

Row 5: Speckle Noise and Row 6: Motion Blur with Columns 1-5 are different algorithms (Column 1: Run RS et al. 2 , Column 2: Ganic E. et al., Column 3:Sverdlov, A. et al., Column 4: Varghese J et al. Column 5: Proposed scheme)

The cropped versions of watermarked images by comparative schemes are given in Fig. 3. From Fig. 3, it is noted that the watermarked outputs of the new scheme although are closely similar with the outputs produced by Varghese J. et al. scheme; it produces better outputs than those of Run RS et al., Ganic E. et al., Sverdlov A. et al. algorithms. 


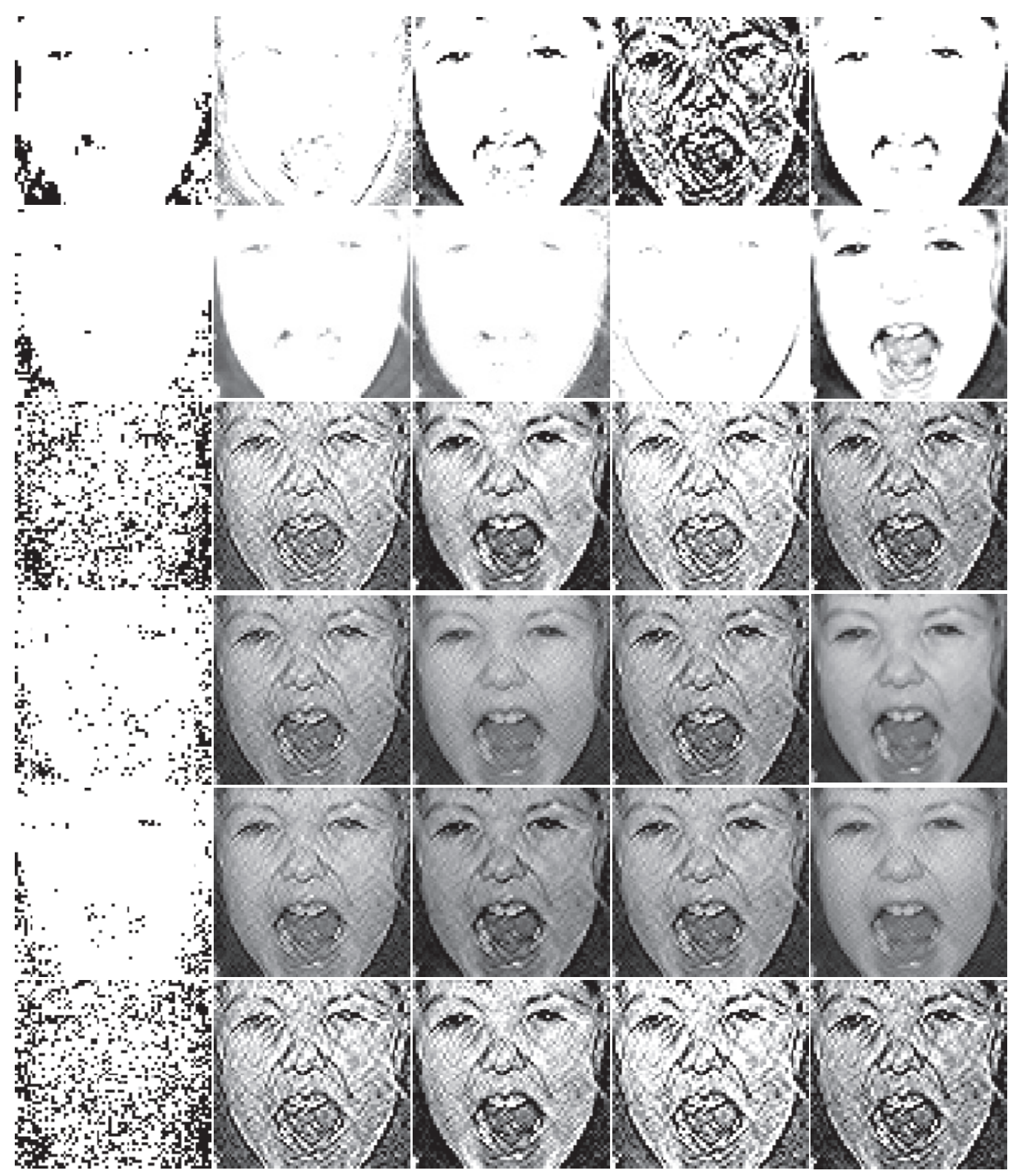

Figure 5. Cropped watermark extracted by comparative schemes from watermarked Scuba image affected by different external attacks: Rows 1-6 are Attacks (Row 1: Unsharp Masking, Row 2: Contrast Stretching, Row 3: JPEG80+Gaussian Noise02, Row 4: JPEG80+SpeckleNoise02, Row 5: JPEG80+Salt\&Pepper02 and Row 6: Gaussian Noise 01+Speckle Noise 05 with Columns 1-5 are different algorithms (Column 1: Run RS et al. 2 , Column 2: Ganic E. et al., Column 3:Sverdlov, A. et al., Column 4: Varghese J et al. Column 5: Proposed scheme)

Table 1 makes the MAE, PSNR, MSSIM, and FSIM analysis of watermarked images by comparative schemes. From Table 1, it is visible that the new method has better PSNR, MSSIM, and FSIM with minimum MAE values than Run RS et al., Ganic E. et al., Sverdlov A. et al., and Varghese J. et al. schemes in most of the cases. 


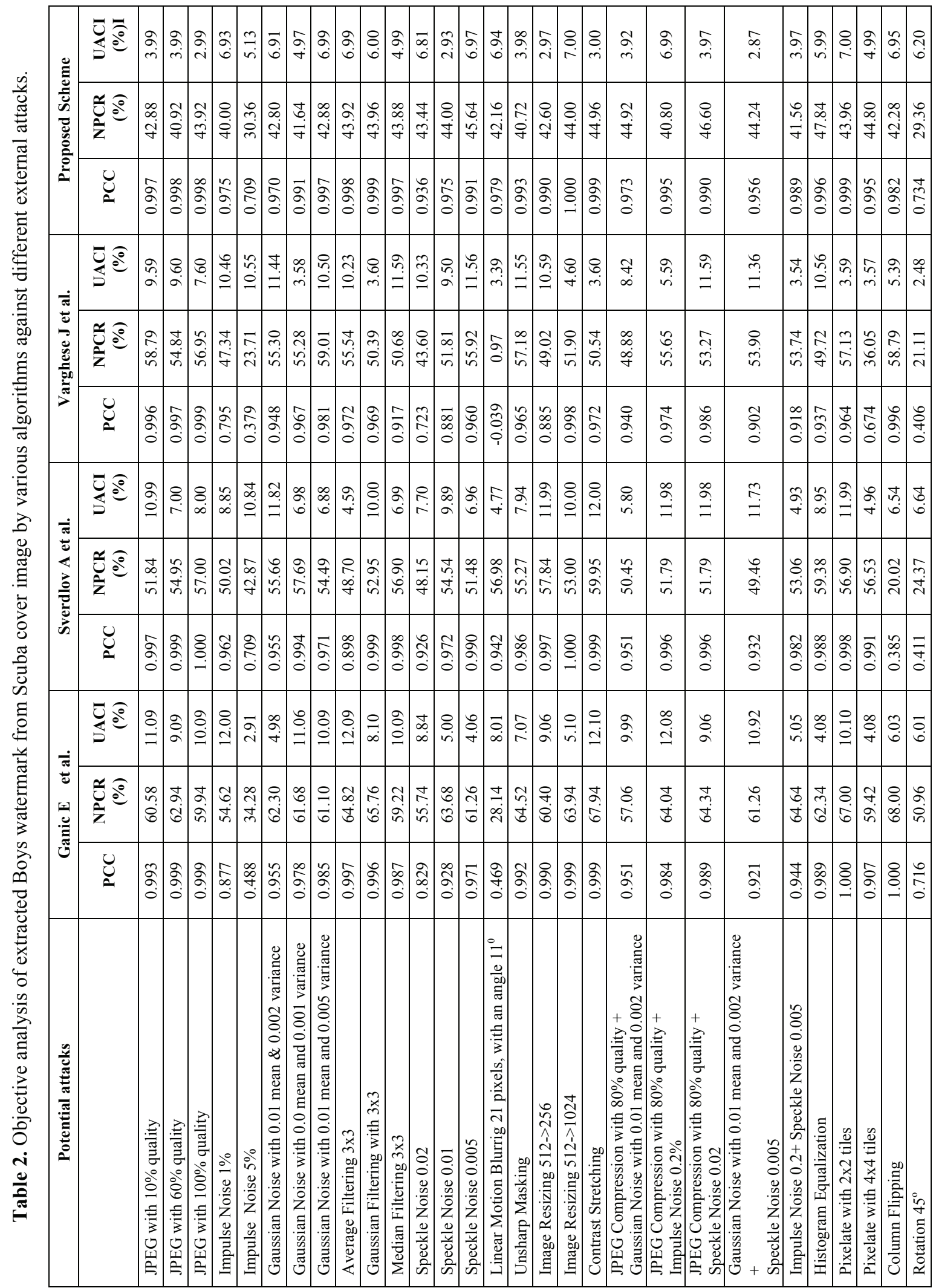




\begin{tabular}{|c|c|c|c|c|c|c|c|c|c|c|c|c|c|c|c|c|c|c|c|c|c|c|c|c|c|c|c|c|c|c|}
\hline & 岩 & $\begin{array}{l}8 \\
7 \\
7\end{array}$ & बे & $\stackrel{8}{\circ}$ & $\begin{array}{l}\infty \\
+ \\
+\end{array}$ & $\overrightarrow{\hat{n}}$ & $\sigma_{\sigma}$ & $\mathbb{1}^{\infty}$ & बे. & 2. & 18 & : & fa & $\begin{array}{c}9 \\
0 \\
0\end{array}$ & coj & $\overrightarrow{5}$ & 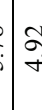 & 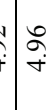 & $\stackrel{8}{8}$ & $\stackrel{\infty}{\stackrel{\infty}{i}}$ & $\begin{array}{l}\infty \\
\infty \\
0 \\
\vdots\end{array}$ & बे & $\vec{\sigma}$ & $\begin{array}{l}\vec{\infty} \\
\dot{r}\end{array}$ & $\mid$\begin{tabular}{|c|} 
\\
$\infty$ \\
$\dot{\infty}$
\end{tabular} & $\begin{array}{l}\mathscr{D} \\
\stackrel{\infty}{+} \\
+\end{array}$ & $\begin{array}{l}\stackrel{0}{\circ} \\
+\end{array}$ & $\hat{\sigma}$ & & ब \\
\hline 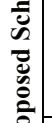 & 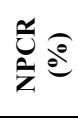 & $\begin{array}{l}+ \\
\infty \\
\text { ले }\end{array}$ & $\begin{array}{l}\tilde{\sigma} \\
\dot{\sigma} \\
\end{array}$ & 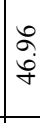 & $\frac{0}{6}$ & $\begin{array}{l}\infty \\
\infty \\
0 \\
\dot{m}\end{array}$ & $\stackrel{\infty}{\stackrel{\infty}{\dot{\sigma}}}$ & 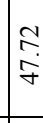 & $\begin{array}{l}\infty \\
\infty \\
\dot{f}\end{array}$ & $\begin{array}{l}\sigma \\
\tilde{y} \\
\gamma\end{array}$ & {$\left[\begin{array}{l}\infty \\
\infty \\
z\end{array}\right.$} & 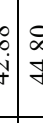 & 象 & $\begin{array}{l}b \\
\dot{b} \\
\vdots \\
\end{array}$ & 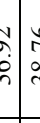 & 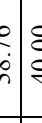 & 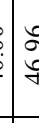 & 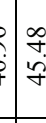 & 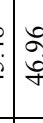 & $\left\{\begin{array}{l}4 \\
\dot{y} \\
\dot{y}\end{array}\right.$ & $\begin{array}{l}\stackrel{J}{J} \\
\stackrel{+}{+}\end{array}$ & $\begin{array}{l}\triangleright \\
\dot{J} \\
\dot{J}\end{array}$ & $\begin{array}{l}\stackrel{+}{\infty} \\
\vec{\forall}\end{array}$ & $\begin{array}{l}\stackrel{q}{+} \\
\underset{j}{j}\end{array}$ & $\mid \begin{array}{l}\infty \\
\infty \\
\infty \\
\infty \\
m\end{array}$ & 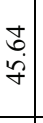 & 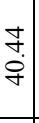 & $\begin{array}{l}\infty \\
0 \\
\dot{j} \\
\dot{f}\end{array}$ & & 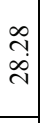 \\
\hline & Uِ & ฉু & ô & ठे & 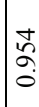 & مै & ลे & ूे & Бू & $\stackrel{\infty}{\circ}$ & 8 & $\dot{d}$ & 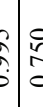 & 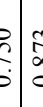 & & ל. & $\frac{8}{0}$ & 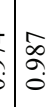 & ठे & ڤ్ & $\begin{array}{l}\overrightarrow{0} \\
\text { oे }\end{array}$ & ڤू & $\vec{a}$ & $\stackrel{\kappa}{\kappa}$ & 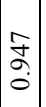 & $\begin{array}{l}\overrightarrow{7} \\
\vdots \\
0\end{array}$ & $\begin{array}{l}\circ \\
\vdots \\
\vdots \\
\vdots\end{array}$ & $\begin{array}{l}\hat{\alpha} \\
\text { o. } \\
\text { o. }\end{array}$ & $\begin{array}{l}0 \\
\stackrel{2}{0} \\
0\end{array}$ & $\tilde{\delta}$ \\
\hline$\pi$ & 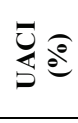 & $\stackrel{\text { : }}{=}$ & in & $\begin{array}{l}\hat{b} \\
\infty \\
\infty\end{array}$ & $\stackrel{\text { f }}{=}$ & $\frac{m}{\infty}$ & $\stackrel{0}{\stackrel{0}{n}}$ & 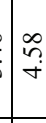 & in & $\stackrel{\square}{i}$ & 4 & 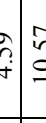 & 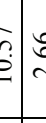 & & 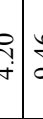 & $\begin{array}{l}0 \\
\vdots \\
\vdots \\
\vdots\end{array}$ & I & $\stackrel{f}{\dot{b}}$ & : & 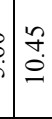 & \begin{tabular}{l}
0 \\
0 \\
0 \\
\hdashline
\end{tabular} & nे & $\begin{array}{l}\stackrel{0}{0} \\
0 \\
0\end{array}$ & $\stackrel{\vec{J}}{\Xi}$ & $\begin{array}{c}\infty \\
m \\
m \\
m\end{array}$ & 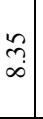 & 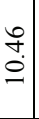 & $\begin{array}{c}\stackrel{\infty}{\rightarrow} \\
\stackrel{+}{+}\end{array}$ & $\begin{array}{l}\text { f } \\
\text { in }\end{array}$ & $\infty$ \\
\hline 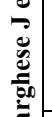 & 总 & 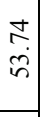 & $\begin{array}{l}8 \\
\dot{0} \\
i\end{array}$ & $\begin{array}{l}8 \\
\text { in }\end{array}$ & $\begin{array}{l}\text { nे. } \\
\text { ले }\end{array}$ & $\begin{array}{l}\hat{\text { a }} \\
\text { ì }\end{array}$ & 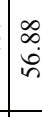 & 角 & $\begin{array}{l}R \\
i n \\
\text { in }\end{array}$ & $\begin{array}{l}0 \\
\text { in } \\
\text { in }\end{array}$ & $\frac{7}{8}$ & $\begin{array}{ll}3 \\
\vdots \\
\vdots\end{array}$ & $\begin{array}{c}0 \\
\dot{b}\end{array}$ & & & 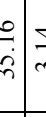 & $\begin{array}{l}0 \\
\infty \\
7 \\
7\end{array}$ & : & {$\left[\begin{array}{l}\infty \\
\infty \\
\infty \\
i n\end{array}\right.$} & 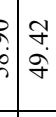 & $\begin{array}{l}\infty \\
\dot{n} \\
i\end{array}$ & $\begin{array}{l}\stackrel{n}{a} \\
\infty \\
\infty \\
i n\end{array}$ & $\begin{array}{l}\infty \\
\dot{n} \\
\dot{n}\end{array}$ & 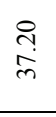 & $\begin{array}{l}\mathbf{b} \\
\dot{m} \\
\dot{m}\end{array}$ & 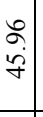 & 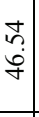 & 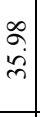 & $\begin{array}{l}\stackrel{+}{+} \\
\dot{+} \\
\text { in }\end{array}$ & i \\
\hline & Uِ & 8 & o. & $\begin{array}{l}\infty \\
\delta \\
\delta\end{array}$ & त्र & 足 & 웅 & 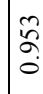 & 气ू. & $\hat{n}$ & $\begin{array}{l}\not \\
\infty \\
0\end{array}$ & $\begin{array}{lll} & 0 \\
0\end{array}$ & $\begin{array}{l}t \\
b \\
b\end{array}$ & a & 5 & $\begin{array}{l}\vdots \\
\vdots \\
0\end{array}$ & $\mid \begin{array}{l}\alpha \\
\infty \\
\infty\end{array}$ & $\begin{array}{l}1 \\
\vdots \\
\vdots \\
\vdots\end{array}$ & $\begin{array}{l}\infty \\
\delta \\
\delta\end{array}$ & 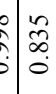 & I & 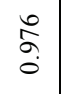 & $\begin{array}{l}\overrightarrow{\widehat{\sigma}} \\
\hat{o}\end{array}$ & $\begin{array}{l}\hat{E} \\
0 \\
0\end{array}$ & $\mid \begin{array}{l}\infty \\
0 \\
0 \\
0\end{array}$ & $\begin{array}{c}0 \\
\vdots \\
\infty \\
0 \\
0\end{array}$ & $\begin{array}{l}\tilde{a} \\
\alpha \\
0 \\
0\end{array}$ & $\begin{array}{l}\infty \\
\hat{n} \\
0\end{array}$ & $\begin{array}{l}2 \\
\check{\sigma} \\
\vdots\end{array}$ & 18 \\
\hline$\dot{\sigma}$ & $\frac{\mathrm{U}}{\mathrm{s}} \mathrm{\varrho}$ & 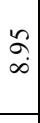 & $\begin{array}{l}\infty \\
\dot{m} \\
\dot{m}\end{array}$ & बे & 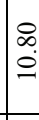 & : & $\stackrel{\infty}{\infty}$ & $\stackrel{\infty}{2}$ & ๙ֶ. & $\stackrel{m}{\stackrel{r}{r}}$ & {$\left[\begin{array}{l}8 \\
\infty\end{array}\right.$} & : & $\dot{r}$ & 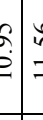 & 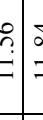 & 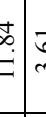 & $\begin{array}{l}\infty \\
\infty \\
\infty\end{array}$ & 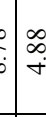 & $: \begin{array}{l}8 \\
\infty\end{array}$ & $\begin{array}{l}\dot{b} \\
\dot{0}\end{array}$ & $\begin{array}{l} \pm \\
\infty \\
i \\
i\end{array}$ & $\begin{array}{l}\infty \\
\infty \\
\infty\end{array}$ & ڤ̊ํ. & $\stackrel{\vec{r}}{\dot{m}}$ & $\begin{array}{l}0 \\
\stackrel{2}{r} \\
\end{array}$ & $\begin{array}{l}\hat{2} \\
\stackrel{0}{0}\end{array}$ & $\begin{array}{l}\dot{1} \\
\stackrel{a}{a} \\
\sigma\end{array}$ & $\begin{array}{l}\vec{t} \\
\stackrel{2}{\sim}\end{array}$ & & $\varepsilon$ \\
\hline 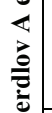 & 艺 & $\left|\begin{array}{l}\infty \\
\tilde{a} \\
i \\
n\end{array}\right|$ & 空 & $\begin{array}{l}\dot{0} \\
\infty \\
i \\
i n\end{array}$ & $\begin{array}{c}\sim \\
\stackrel{\sim}{i} \\
\sim\end{array}$ & 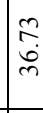 & 官 & 㝒 & $\stackrel{\text { If }}{\stackrel{f}{n}}$ & \begin{tabular}{l}
$\tilde{\sigma}$ \\
\multirow{2}{*}{}
\end{tabular} & $\mid \begin{array}{l}a \\
\infty \\
\infty \\
v\end{array}$ & \begin{tabular}{l|l}
$\vdots$ \\
$\vdots$ \\
0 \\
$\vdots$
\end{tabular} & 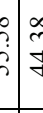 & & $\begin{array}{c}\infty \\
\substack{y \\
y} \\
\vdots\end{array}$ & $\begin{array}{lll}5 \\
\vdots \\
\vdots & 8\end{array}$ & $\begin{array}{l}8 \\
0 \\
i n\end{array}$ & $\begin{array}{l}b \\
b \\
b\end{array}$ & 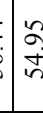 & 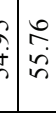 & 5 & 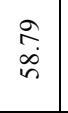 & $\begin{array}{l}\stackrel{9}{+} \\
\dot{+} \\
\dot{n}\end{array}$ & $\begin{array}{l}\stackrel{+}{\mathbf{H}} \\
\stackrel{n}{n}\end{array}$ & $\mid \begin{array}{l}0 \\
\infty \\
\dot{q} \\
\dot{\sigma}\end{array}$ & 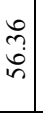 & $\begin{array}{l}a \\
\text { ڤे } \\
\text { nे }\end{array}$ & 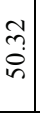 & 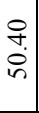 & | \\
\hline & U. & $\begin{array}{l}\infty \\
\infty \\
\infty\end{array} \mid$ & o̊ & oे & ơ & हैं & d. & वे & $\begin{array}{c}\infty \\
\stackrel{0}{0} \\
\delta\end{array}$ & $\begin{array}{c}\hat{0} \\
\tilde{\infty} \\
0\end{array}$ & 8 & : & c. & 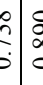 & 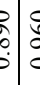 & 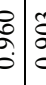 & $\frac{7}{8}$ & : & हे & s. & 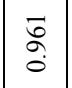 & ڤे & $\begin{array}{l}\stackrel{\circ}{\circ} \\
\stackrel{\circ}{\circ}\end{array}$ & 今े & ڤે & $\begin{array}{l}0 \\
\grave{o} \\
\vdots\end{array}$ & $\begin{array}{l}0 \\
: \\
0\end{array}$ & $\begin{array}{l}\stackrel{0}{a} \\
\text { o. }\end{array}$ & & $\mid$ \\
\hline & 式 & $\mid$\begin{tabular}{l}
0 \\
\hdashline \\
\end{tabular} & 宓 & ठे. & $\stackrel{\infty}{\stackrel{\infty}{r}}$ & $\underset{\text { I. }}{\stackrel{2}{r}}$ & $\underset{\infty}{\infty}$ & {$\left[\begin{array}{l}\infty \\
\stackrel{0}{\ominus}\end{array}\right.$} & 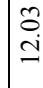 & $\stackrel{f}{\stackrel{f}{+}}$ & i & 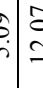 & is & 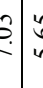 & $\begin{array}{l}6 \\
\dot{r}\end{array}$ & is & $\mid \begin{array}{l}\infty \\
\alpha \\
\gamma\end{array}$ & $\stackrel{\substack{f \\
\dot{f}}}{\stackrel{\leftrightarrow}{+}}$ & $\frac{0}{\infty}$ & 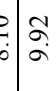 & 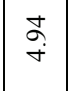 & $a$ & $\stackrel{n}{\stackrel{g}{\Xi}}$ & $\begin{array}{l}\infty \\
\infty \\
i\end{array}$ & $\mid \begin{array}{l}0 \\
\infty \\
0 \\
0\end{array}$ & $\mid$ & $\stackrel{t}{i}$ & $\stackrel{\Re}{\sim}$ & & $\mid \begin{array}{l}\alpha \\
\dot{a} \\
\gamma\end{array}$ \\
\hline$\stackrel{0}{\varrho}$ & 艺 & $\frac{q}{q}$ & $\begin{array}{l}2 \\
0 \\
\dot{8} \\
0\end{array}$ & $\begin{array}{l}\infty \\
\infty \\
\dot{b}\end{array}$ & $\begin{array}{l}\text { ì } \\
\text { in }\end{array}$ & ปี & $\frac{0}{\mathrm{i}}$ & $\stackrel{ \pm}{\dot{J}}$ & $\begin{array}{l}\circ \\
\stackrel{0}{0} \\
\stackrel{0}{0}\end{array}$ & $\begin{array}{l}\text { \& } \\
\text { तु }\end{array}$ & 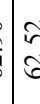 & \begin{tabular}{c|c}
$\tilde{c}$ & $\delta$ \\
\hdashline & $\delta$
\end{tabular} & ¿े & 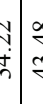 & 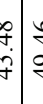 & \begin{tabular}{l|l}
0 \\
$\stackrel{f}{*}$ \\
$g$
\end{tabular} & {$\left[\begin{array}{l}\infty \\
\infty \\
1 \\
b\end{array}\right.$} & 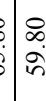 & $\begin{array}{l}t \\
0 \\
0\end{array}$ & 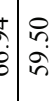 & $\begin{array}{l}\infty \\
\stackrel{2}{\circ} \\
\text { nे }\end{array}$ & 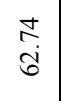 & $\begin{array}{l}\infty \\
\stackrel{\infty}{8} \\
\stackrel{8}{0}\end{array}$ & 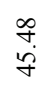 & $\begin{array}{l}2 \\
\stackrel{2}{0} \\
\dot{f}\end{array}$ & $\begin{array}{l}\vec{b} \\
\dot{b} \\
\dot{b}\end{array}$ & $\begin{array}{l}0 \\
\dot{8}\end{array}$ & & $\begin{array}{l}8 \\
8 \\
8\end{array}$ & $\begin{array}{l}8 \\
0 \\
0 \\
0\end{array}$ \\
\hline & Uِ & ڤ. & ô. & o. & $\stackrel{i}{i}$ & s. & 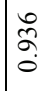 & \&े & $\begin{array}{l}\bar{\infty} \\
\stackrel{8}{\circ}\end{array}$ & : & \& & d. & $\begin{array}{lll}0 \\
0\end{array}$ & $\hat{c}$ & 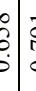 & $\begin{array}{ll}5 \\
\delta \\
5\end{array}$ & $\begin{array}{l}\mathscr{0} \\
0 \\
0\end{array}$ & $\begin{array}{l}b \\
\vdots \\
\vdots \\
\dot{b}\end{array}$ & के & 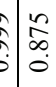 & $\begin{array}{c}\tilde{o} \\
\hat{\sigma} \\
\hat{o}\end{array}$ & ڤે̀ & $\stackrel{\infty}{\grave{o}}$ & 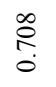 & $\mid \begin{array}{l}0 \\
\stackrel{1}{0} \\
0\end{array}$ & 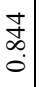 & $\begin{array}{l}2 \\
\infty \\
0 \\
0\end{array}$ & 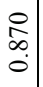 & & $\bar{a}$ \\
\hline 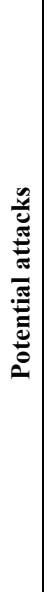 & & 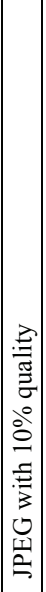 & 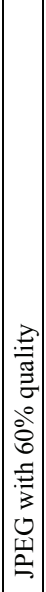 & 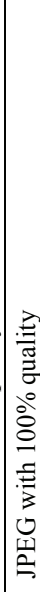 & 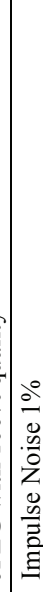 & $\begin{array}{l}0 \\
\text { in } \\
0 \\
\frac{0}{2} \\
z \\
0 \\
0 \\
0\end{array}$ & 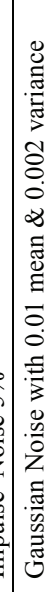 & 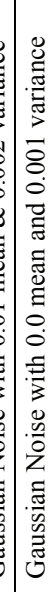 & 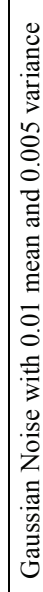 & 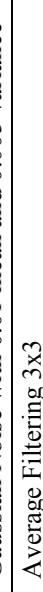 & 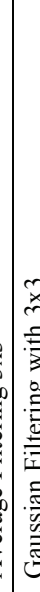 & 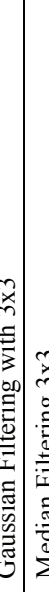 & 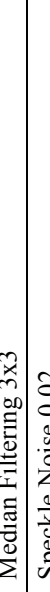 & 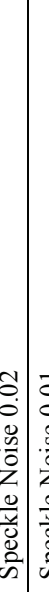 & 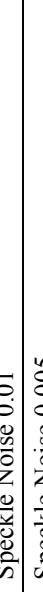 & 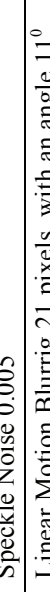 & 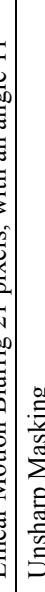 & 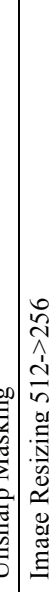 & 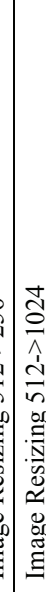 & 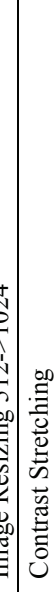 & 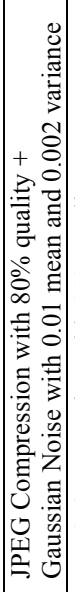 & 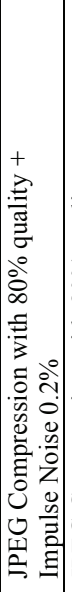 & 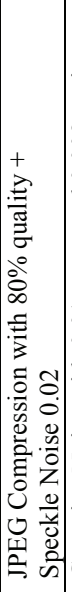 & 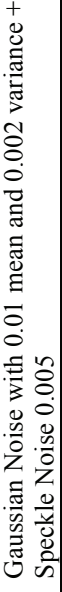 & 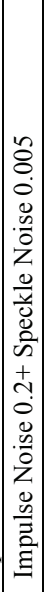 & 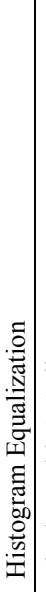 & 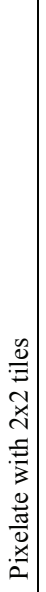 & 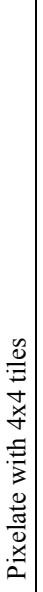 & 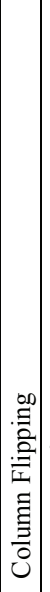 & \\
\hline
\end{tabular}




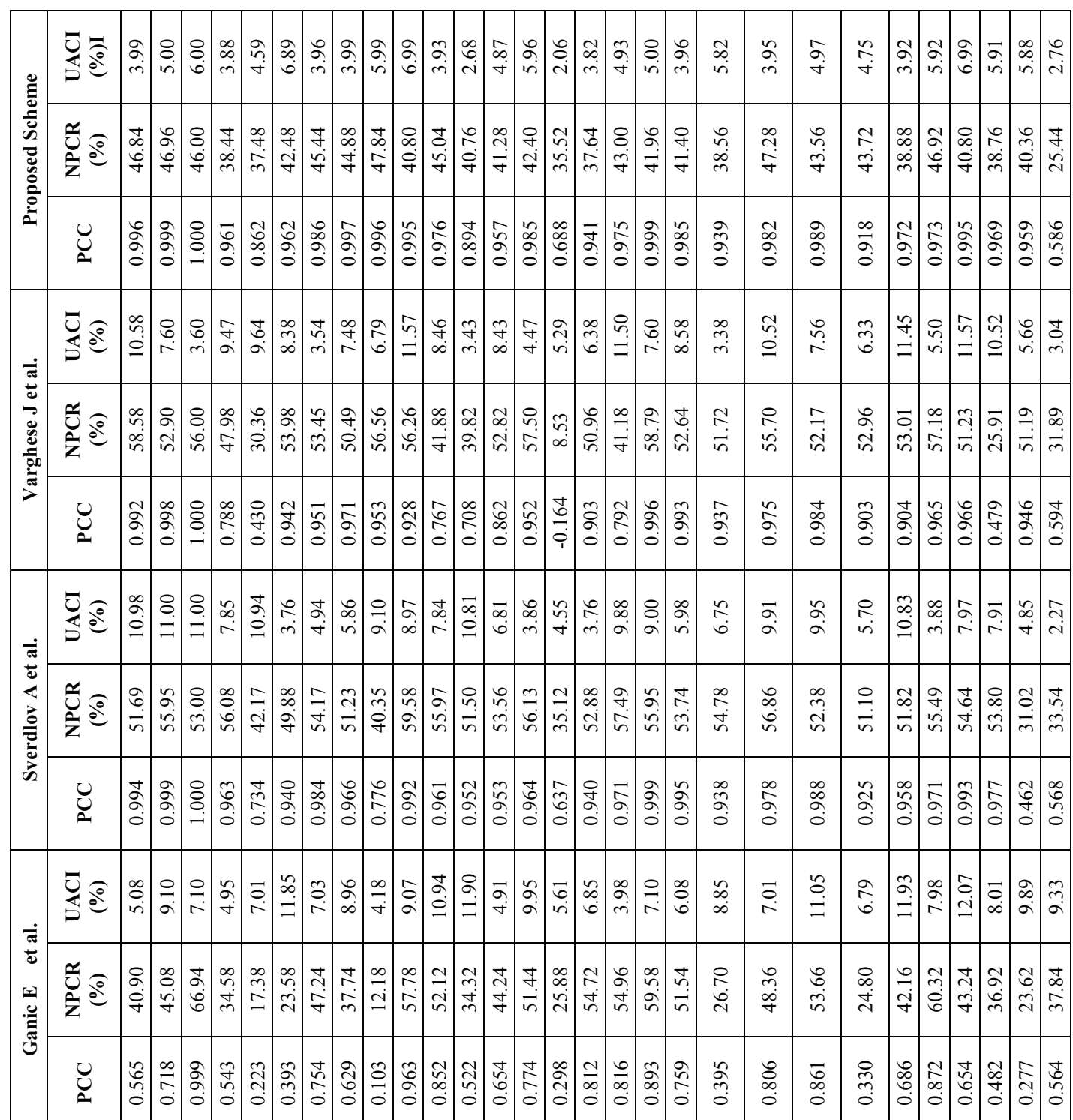


Robustness analysis of different algorithms is performed in terms of Pearson's Correlation coefficient (PCC), Number of Changing Pixel Rate (NPCR), and the Unified Averaged Changed Intensity (UACI) (Wu, Y. et al., 2011). PCC quantifies the linear associative relationship between singular values of original and the extracted watermarks (Poljicak et al., 2011). PCC outcome varies from +1 to -1 . If PCC outcome is +1 , it indicates maximum positive linear correlation, while 0 indicates noncorrelation among two singular value matrices. Also, -1 indicates a highly negative linear correlation showing large deviation between the singular values of the original and extracted watermarks. For conducting effective comparison of all algorithms, the best PCC values from all watermark replicas are used for assessing the robustness of all algorithms used in the study. In cryptography, NPCR/UACI scores are usually used for analyzing the resistance of cipher images to the changes in pixels of plain images. The Number of Changing Pixel Rate (NPCR) and the Unified Averaged Changed Intensity (UACI) determine the robustness of image watermarking against external potential attacks. Although higher NPCR/UACI score normally shows higher resistance to differential attacks in the case of cipher and plain texts, but for watermarking, a lower NPCR/UACI score indicates higher robustness of watermarking schemes against external potential attacks. If $C W$ and $C W$ denote the original and the extracted watermarks, the NCPR is defined as

$$
N C P R=\frac{\sum_{i=1}^{M} \sum_{j=1}^{N} D(i, j)}{M \times N} \times 100 \quad(\%)
$$

where

$$
D(i, j)= \begin{cases}1 & \text { if } C W(i, j)=\tilde{C W}(i, j) \\ 0 & \text { Otherwise }\end{cases}
$$

The UACI is defined by

$$
U A C I=\frac{\sum_{i=1}^{M} \sum_{j=1}^{N}|C W(i, j)-\tilde{C W}(i, j)|}{M \times N^{*} 255} \times 100(\%)
$$

The average bitrate for all algorithms is kept constant to analyze the robustness of all algorithms. The external attacks used for analyzing the robustness of different algorithms are JPEG, impulse noise, Gaussian noise, average filtering, Gaussian filtering, median filtering, speckle noise, motion blurring, unsharp masking, image resizing, contrast stretching, histogram equalization, pixelate, flipping, and rotation. PCC, NPCR, and UACI values of different algorithms obtained for different watermark and carrier images damaged with various potential attacks are presented in Table 2 to Table 4.

From Table 2 to Table 4, it is found that the new algorithm is consistent in its performance for majority of attacks. The higher PCC with lower NPCR and UACI values produced by the proposed scheme for most of the 
external attacks demarcate the consistency and improved performance of new scheme over other comparative algorithms used in the experimental study. Fig. 4 and 5 show the cropped versions of extracted Boys watermarks produced by Run RS et al., Ganic E. et al., Sverdlov A. et al., Varghese J. et al. and the proposed algorithms from watermarked Scuba images. From Fig. 4 and 5, it is clear that the new scheme is effective in resisting JPEG80, speckle noise, and filtering based potential attacks, while its performance is moderate against Gaussian noise and contrast stretching based potential attacks.

\section{CONCLUSION}

The paper introduced a new watermarking algorithm by attaching replicas of watermark contents in carrier image by combining the advantages of DWT, DFT, DCT, and SVD transformations. As the proposed algorithm attaches multiple replicas in different frequencies of host image, the proposed algorithm provided more opportunity to extract at least some undamaged copies of watermark when potential attacks affect other attached frequencies. By combining similar frequency subbands at different levels using different frequency transformations, the algorithm refines the frequency bands and thereby paves the way for providing better robustness for attaching watermark components in case of potential attacks. Image fidelity of watermarked images generated by comparative algorithms is evaluated in terms MAE, PSNR, MSSIM, and FSIM. The experimental analysis based on visual and quantitative metrics such as NPCR and UACI with wide set of test images showed that the robustness of the new scheme is consistent for all attacks, and it provided better visual output with improved quantitative metrics than other comparative algorithms for most of the external attacks.

\section{REFERENCES}

Borra, S., Thanki, R., \& Dey, N. 2018. Digital image watermarking: theoretical and computational advances. CRC Press.

Ganic, E., \& Eskicioglu, A. M. 2004, September. Robust DWT-SVD domain image watermarking: embedding data in all frequencies. In Proceedings of the 2004 Workshop on Multimedia and Security (pp. 166-174). ACM.

Hsu, C. S., \& Tu, S. F. 2019. Enhancing the robustness of image watermarking against cropping attacks with dual watermarks. Multimedia Tools and Applications, 1-27.

Hu, H. T., Hsu, L. Y., \& Chou, H. H. 2020. An improved SVD-based blind color image watermarking algorithm with mixed modulation incorporated. Information Sciences.

Hussain, O. B., Razak, A., \& Varghese, J. 2019. Comparative Analysis of Prominent Watermarking Algorithms. In Innovations in Computer Science and Engineering (pp. 361-369). Springer, Singapore.

Ko, H. J., Huang, C. T., Horng, G., \& Shiuh-Jeng, W. A. N. G. 2020. Robust and blind image watermarking in DCT domain using inter-block coefficient correlation. Information Sciences, 517, 128-147.

Li, J., Yu, C., Gupta, B. B., \& Ren, X. 2018. Color image watermarking scheme based on quaternion Hadamard transform and Schur decomposition. Multimedia tools and applications, 77(4), 4545-4561.

Mehta, R., Rajpal, N., \& Vishwakarma, V. P. 2018. Robust image watermarking scheme in lifting wavelet domain using GA-LSVR hybridization. International Journal of Machine Learning and Cybernetics, 9(1), 145-161.

Najafi, E., \& Loukhaoukha, K. 2019. Hybrid secure and robust image watermarking scheme based on SVD and sharp frequency localized contourlet transform. Journal of information security and applications, 44, 144156. 
Navas, K. A., Ajay, M. C., Lekshmi, M., Archana, T. S., \& Sasikumar, M. 2008, January. Dwt-dct-svd based watermarking. In 2008 3rd International Conference on Communication Systems Software and Middleware and Workshops (COMSWARE'08) (pp. 271-274). IEEE.

Nematollahi, M. A., Vorakulpipat, C., \& Rosales, H. G. 2017. Digital watermarking. Springer Singapore.

Poljicak, A., Mandic, L., \& Agic, D. 2011. Discrete Fourier transform-based watermarking method with an optimal implementation radius. Journal of Electronic Imaging, 20(3), 033008.

Run, R. S., Horng, S. J., Lai, J. L., Kao, T. W., \& Chen, R. J. 2012. An improved SVD-based watermarking technique for copyright protection. Expert Systems with applications, 39(1), 673-689.

Sharma, D., Saxena, R., \& Singh, N. 2017. Dual domain robust watermarking scheme using random DFRFT and least significant bit technique. Multimedia Tools and Applications, 76(3), 3921-3942.

Singh, A. K. 2017. Improved hybrid algorithm for robust and imperceptible multiple watermarking using digital images. Multimedia Tools and Applications, 76(6), 8881-8900.

Su, Q., Liu, D., Yuan, Z., Wang, G., Zhang, X., Chen, B., \& Yao, T. 2019. New Rapid and Robust Color Image Watermarking Technique in Spatial Domain. IEEE Access, 7, 30398-30409.

Sverdlov, A., Dexter, S., \& Eskicioglu, A. M. 2006. Secure DCT-SVD domain image watermarking: embedding data in all frequencies. In Image Processing Seminar at Brooklyn College, NY.

Thakur, S., Singh, A. K., Kumar, B., \& Ghrera, S. P. 2020. Improved DWT-SVD-Based Medical Image Watermarking Through Hamming Code and Chaotic Encryption. In Advances in VLSI, Communication, and Signal Processing (pp. 897-905). Springer, Singapore.

Umamageswari, A., \& Suresh, G. R. 2014. Secure medical image communication using ROI based lossless watermarking and novel digital signature. Journal of Engineering Research, 3(2), 1-22.

Varghese, J., Subash, S., Hussain, O. B., Nallaperumal, K., Saady, M. R., \& Khan, M. S. 2016. An improved digital image watermarking scheme using the discrete Fourier transform and singular value decomposition. Turkish Journal of Electrical Engineering \& Computer Sciences, 24(5), 3432-3447.

Varghese, J., Subash, S., Hussain, O. B., Saady, M. R., Babu, B., \& Riazuddin, M. 2015. Image adaptive DCT-SVD based digital watermarking scheme by human visual characteristics. Journal of Engineering Research, 1(3), 1-18.

Varghese, J., Omer B. H., Bijoy B., Jamshid M. B., Saudia S., Mohamed R. S., and Mohamed S. K.. 2014. An efficient DCT-SVD based algorithm for digital image watermarking. In 2014 International Carnahan Conference on Security Technology (ICCST), (pp. 1-6). IEEE.

Wang, C., Wang, X., Xia, Z., \& Zhang, C. 2019. Ternary radial harmonic Fourier moments based robust stereo image zero-watermarking algorithm. Information Sciences, 470, 109-120.

Wu, Y., Noonan, J. P., \& Agaian, S. 2011. NPCR and UACI randomness tests for image encryption. Cyber journals Multidisciplinary journals in science and technology, Journal of Selected Areas Telecommunications (JSAT), 1, (2), 31-38.

Yadav, B., Kumar, A., \& Kumar, Y. 2018. A robust digital image watermarking algorithm using DWT and SVD. In Soft Computing: Theories and Applications (pp. 25-36). Springer, Singapore. 